\title{
Identification and characterization of metastasis- associated individualized gene expression signature in osteosarcoma
}

Guofeng Zhang ( $\nabla$ gfzhangknu@gmail.com )

Yantai Affiliated Hospital of Binzhou Medical University

Yonglin Zhu

Yantai Affiliated Hospital of Binzhou medical University

Chengzhen Jin

Yantai Affiliated Hospital of Binzhou Medical University

Baisui Zhou

Yantai Affiliated Hospital of Binzhou Medical University

Shanrun Zhao

Yantai Affiliated Hospital of Binzhou Medical University

Chuanbo Li

Yantai Affiliated Hospital of Binzhou Medical University

\section{Fangang Fu}

Yantai Affiliated Hospital of Binzhou Medical University

Shanhui Li

Yantai Affiliated Hospital of Binzhou Medical University

\section{Research article}

Keywords: metastasis; gene expression; survival; biomarker; osteosarcoma

Posted Date: March 26th, 2020

DOI: https://doi.org/10.21203/rs.3.rs-18944/v1

License: (c) (1) This work is licensed under a Creative Commons Attribution 4.0 International License. Read Full License 


\section{Abstract}

Introduction: Osteosarcoma (OS) patients with complete surgical resection still relapse with poor prognosis. Part of this is due to the inability to accurately detect distant metastasis. Thus it's enssential to identify metastasis-related biomarkers for OS.

Methods: In present study, a computational pipeline based on relative expression orderings (REOs) using gene expression proles was constructed in metastases and non-metastases OS patients.

Results: 138 metastasis-associated gene pair signature (MGPS) were identified follow two independent datasets. In order to further extract metastasis-associated biomarker for clinical application, a metastases-specific co-expressed MGPS network was constructed and analyzed. MGPS such as MYL5 and RPL27A showed strong positive correlation (Cor $=0.75, \mathrm{P}<0.001)$ in metastatic OS patients. There were thirteen MGPSs in above network were associated with prognosis. These prognostic MGPSs could become as a specific classifier to distinguish metastases and non-metastases OS patients. Functional analysis showed MGPSs were associated with cancer metastasis-related functions. Drug and MGPS network could provide some drug candidates for treatment of OS.

Conclusions: Collectively, the roles of the MGPSs in OS were elucidated, which could be beneficial for understanding OS pathogenesis and treatment.

\section{Background}

The American Cancer Society reported that the incidence of childhood and adolescent cancers is increasing rapidly. Among these, osteosarcoma (OS) is one of the cancer with highest mortality rate in the paediatric population [1]. In the last 35 years, there was no significant improvement in the five-year survival rate if metastases are present during diagnosis [2,3]. Similar to the observation in other cancers, metastasis is a major factor fo most death (75\%) of OS patients [4]. For non-metastatic OS patients, tumor resection together with chenotherapy is a successful treatment plan. However, planning appropriate treatment for metastatic OS is still a great challenge for researchers and clinicians.

Cancer metastasis includ two process: (1) cancer cells disseminate from primary tumors to whole body. (2) establish secondary lesions in distant organs [5]. Identifying metastases-associated biomarker was a key insight of diagnosis and treatment for OS. Recent years, some attempt has been contributed to identify metastases-associated biomarkers using gene expression profiles for many kinds of cancers [68]. However, the number of metastases-associated biomarkers which could be applicated for clinic is very small. Usually, most of the suggested metastases-associated biomarkers, classify patients into different risk groups by differential expression or clustering. The thresholds of differential expression which generated from training data set could't be directly applied to another data set. One of major reason is the gene expression level is sensitve based on diverse batch effect and platform of microarray. Furthermore, this means that the generated data of all the samples detected together should be normalized when only 
deal with a single sample. In other words, risk composition of other samples which are normalized together determines the risk prediction of a single sample [9].

One of insensitive approach for systematic biases of microarray is within-sample relative expression orderings (REOs). REOs are invariant and robust for monotonic data normalization and against differences within biological indibiduals $[9,10]$. In past years, REOs have been proposed to identify prognostic signatures $[11,12]$. However, the performance of REOs in predicting metastases-associated signatures for OS has been not reported. Therefore, it is worth tring to identify robust metastasesassociated biomarkers for clinical application in OS based on the rank-based approach.

In present study, a computational pipeline based on REOs using gene expression proles was constructed in metastases and non-metastases OS patients. Follow two independent datasets, 138 common metastasis-associated gene pair signature (MGPS) were identified in OS. A metastases-specific coexpressed MGPS network and its topological characteristics was constructed and analyzed. Some of MGPS such as MYL5 and RPL27A showed strong positive correlation (Cor $=0.75, P<0.001$ ) in metastatic OS patients. Thirteen prognostic MGPSs in co-expressed MGPS network were discovered. These prognostic MGPSs could better classify metastases and non-metastases OS patients. Functional analyses suggested that MGPSs were related with regulation of cancer and cancer-related pathways. Drug and MGPS network could provide some drug candidates for treatment of OS. Collectively, this study could provide assistance for diagnosis and treatment of OS.

\section{Results}

\section{Extract GMPSs in OS for metastases and non-metastases patients}

We built an integrated and computational pipeline based on REO approach using gene expression profiles (Figure 1A). First, 245 metastases-related genes were identified and they would be used to follow analysis. Second, a 0-1 matrix for a pair of genes $\left(G_{m}\right.$ and $\left.G_{n}\right)$ in metastases and non-metastases OS patients according to expression pattern of the gene pair. Then, if the frequency of metastases OS patients accord with a particular REO pattern was measured by fisher's exact test compared with nonmetastases controls. The particular REO pattern was expression $\mathrm{Gm}>\mathrm{Gn}$ or $\mathrm{Gm}<\mathrm{Gn}$. Lastly, MGPSs were identified if the $P$ values of Fisher's exact test were smaller than 0.05 . We performed this pipeline based on dataset from TARGET. The distribution of P values of MGPSs with fluctuation is between 0.01 and 0.05 (Figure 1B). We divide all $P$ values into five regions: 0 to $0.01,0.01$ to $0.02,0.02$ to $0.03,0.03$ to 0.04 and 0.04 to 0.05 . The amount in each region had no great difference (Figure $1 \mathrm{C}$ ). In order to ensure the accuracy and stability of MGPSs for OS, another dataset GSE33382 from GEO was used for identifying MGPSs. The $P$ values of GSE33382 showed similar distribution to TARGET dataset (Figure 1D, 1E). Lastly, we got 138 common MGPSs in both dataset TARGET and GSE33382 for OS (Figure 1F).

\section{Some MGPSs showed strong correlation in metastases-specific co-expressed network for OS}


The correlation values of MGPSs would showed great difference between metastases and nonmetastases OS patients. According to the inference, a metastases-specific co-expressed network was constructed (Figure 2A). The network contained 71 nodes and 57 edges. More than half MGPSs showed great difference (absolute values of difference for PCCs $>0.25$ ) between metastases and non-metastases OS patients. There were $50.88 \%$ and $5.26 \%$ MGPSs were both positive and negative correlated in metastases and non-metastases OS patients (Figure 2B). The correlated direction of $43.86 \%$ MGPSs were reversed in metastases and non-metastases OS patients. The global network showed scale-free distribution (R square $=0.75$ ) which is a specific topological feature of transcriptional regulatory network (Figure 2C). We found metastases-related gene RPL27A had highest degree in this metastases-specific co-expressed network (degree= 19). Most of the interacted strength between RPL27A and other genes had great difference in metastases and non-metastases OS patients. For example, gene RPL27A and MYL5 had strong positive correlations (Cor= $0.75, \mathrm{P}<0.001$ ) in metastatic OS patients (Figure 2D). However, gene RPL27A and MYL5 were not correlated in non-metastatic OS patients (Cor $=0.02, P=0.86$, Figure 2E). The results indicated that these MGPSs maybe play key and specific roles in metastases process for OS patients.

\section{Some genes in MGPSs were associated with prognosis for OS patients}

As we known, time to metastases and number of lesions are the most important prognostic factors for OS patients [13]. Thus it's essential to consider the prognostic roles of MGPSs in OS patients. 13 genes were associated with survival in above metastases-specific co-expressed network for OS (Figure 3A). For example, prognosis-related MGPSs CYBB and P4HA1 showed strong negative correlations in metastases OS patients (Cor $=-0.35$, Figure 3B). Prognosis-related MGPSs CYBB and P4HA1 also showed strong negative correlations in metastases OS patients (Cor $=-0.25$, Figure $3 C$ ). Gene P4HA1, CYBB and CD53 were all related with survival (Figure 3D, E, F). In addition, samples with high expression for these genes usually showed worse survival. All the results indicated that some genes in MGPSs were associated with prognosis for OS patients.

\section{Prognosis-related genes in MGPSs could classify metastases and non-metastases OS patients}

We constructed a prognosis-related MGPS network which extracted from metastases-specific coexpressed network. This network contained prognosis-related genes and their direct neighbored genes in metastases-specific co-expressed network (Figure 4A). There were 25 nodes (13 prognosis-related genes) and 18 edges in this prognosis-related MGPS network. We found three MGPSs including P4HA1-CYBB, P4HA1-EVI2B and P4HA1-CD53 were all associated with survival. In order to validate if these prognosis genes in MGPSs could become biomarkers for metastases of OS patients, we classify metastases and non-metastases OS patients using gene expression profile follow a consensus clustering method. The prognosis-related genes in MGPSs could distinguish all samples into diverse groups. Final number of group was 4 based on area under CDF curve plot (Figure 4B and C). Each OS patients group had a consensus expression pattern and could distinguish clearly (Figure 4D). Most OS patients could be classified accurately (Chi-square test, $P<0.001$ ) and especially the last group (C4) were all matched with 
the non-metastases OS patients (Figure 4E). Collectively, all the above results indicated that the expression of prognosis-related genes in MGPSs could be served as specific biomarkers to distinguish metastases and non-metastases OS patients.

\section{Functional characterizations and drug repurposing candidates of MGPSs in OS}

Functional enrichment analyses were performed to characterize the functions of MGPSs in OS. For GO enrichment analyses, these MGPSs were enrichment in some critical biological functions such as regulation of microtubule polymerization, negative regulation of autophagy and negative regulation of smooth muscle cell migration (Figure 5A). Dynamics of actin filament cooperated with microtubules could drive cell motility process. Many studies revealed that microtubule dynamics were necessary for promoting epithelial-mesenchymal transition (EMT) [14-16]. Expansion of dormant tumor cells into metastases or anti-tumor inflammatory responses would be restricted and promoted by autophagy. On the contrary, metastasis would be promoted by self-eating based on strengthening fitness of tumor cell environmental stresses response including anoikis during metastatic progression [17]. For KEGG pathway enrichment analyses, these MGPSs were enrichment in some key pathways associated with cancer or metastasis such as Natural killer cell mediated cytotoxicity, B cell receptor signaling pathway and MAPK signaling pathway (Figure 5B). Natural killer cells participate to immune response against metastasis [18]. These functional enrichment analysis showed these GMPSs were associated with cancer metastasis.

We also constructed a drug-related MGPS network to explore drug repurposing candidates for OS (Figure 5C). We found Proline (DB00172) was a drug repurposing candidate and its target gene were P4HA1 and PYCR2. Proline is one of the twenty amino acids in organism, which is a component of protein. Normal functions of jonts and tendons and maintain and strengthen all reply on proline at a great extent [19]. Azacytidine is clinically used to treat myelodysplastic syndrome, a group of heterogeneous bone marrow stem cell diseases [20]. In our analysis, Azacitidine was considered as a drug candidate for OS.

Azacitidine is a cytidine nucleoside analogue, which has the clinical activity of myelodysplastic syndrome and the potential activity of solid tumor.

\section{Discussion}

In this study, we suggested that the MGPSs using REO approach follow expression level of genes play specific roles in metastasis process of OS patients. These MGPSs could be as metastasis-related biomarkers for OS. Differential expression analysis for gene expression profiles between metastases and non-metastases samples was considered as a common approach. The adoption of normalization for other samples contribute to differential expression of patients and it would generate a lot of uncertainty for risk classification of patient. Especially, the uncertainty would increase if the sample size was small and could't represent all the disease patients [21]. Specially, qualitative REOs-based MGPSs would identify more accurated personalized information of individual patient than traditional differential expression in clinical application. 
A hypothesis that the OS patients with poor prognosis might harbor metastases was advanced. Thus we performed survival analysis and identified 13 prognosis-related genes in MGPSs. The problems of nsufficient power and too complex to evaluate metastasis would be present based on all the MGPSs in OS patients. According the clinical needs, we proposed prognosis-related MGPSs based a strict voting criterion for survival analysis and proved that these prognosis-related MGPSs performed better than all the MGPSs. The results indicated that prognosis-related MGPSs maybe more suitable for clinical application of predicting metastasis. In order to explore if these prognosis-related genes in MGPSs could be specific biomarkers of metastasis in OS patients, we used them to classify metastases and nonmetastases patients based on gene expression. The 13 prognosis-related genes could classify metastases and non-metastases patients $(P<0.001)$.

In prognosis-related GMPSs network, gene P4HA1 was a key node with highest degree. The previous study has reported that P4HA1 was the active catalytic component of prolyl 4-hydroxylase in cancers [22], glioma [23], prostate cancer [24] and pancreatic cancer [25], P4HA1 can promote chemoresistance, tumor growth and metastasis. The prognosis-related gene CD53 could form a MGPS with P4HA1 in OS patients. CD53 is essential for CD2 signal transduction, growth regulation and cell survival of cancer [26]. P4HA1 was also a drug target in drug-related MGPS network. The roles of P4HA1 in OS should be explored and validated.

\section{Conclusions}

In this study, some MGPSs were identified and characterized for OS patients based on gene expression profiles. Correlations of some MGPSs showed obvious difference between metastases and nonmetastases samples. Prognosis-related genes in metastases-specific co-expressed network could become as specific biomarkers to classify metastases and non-metastases OS patients. The functional analysis showed the association between MGPSs and cancer metastasis in OS patients. Collectively, our study provides novel insights into the mechanisms underlying the roles of MGPSs in metastasis process for OS.

\section{Materials And Method}

\section{Clinical and gene expression profile datasets of OS}

There were two main independent datasets in present study. First dataset including gene expression and related clinical information of 92 OS patients containing 23 and 69 metastatic and non-metastatic patients were obtained from the Therapeutically Applicable Research To Generate Effective Treatments (TARGET, https://ocg.cancer.gov/programs/target) data portal, which included 17070 mRNAs. TARGET program provides a conprehensive genomic landscape to explore molecular characteristics of childhood cancers. Providing a novel guide for developing effective therapeutic plans based on generated data is the major goal of TARGET program. The case selection criteria and sample details could be obtained at https://ocg.cancer.gov/programs/target/projects/osteosarcoma. Then, the second gene expression 
profile was obtained from gene expression omnibus (GEO, https://www.ncbi.nlm.nih.gov/geo/query/acc.cgi?acc=GSE33382). Dataset GSE33382 gene expression profile included 23 and 69 metastatic and non-metastatic patients. The detailed information of patients could be found in previous study $[27,28]$. Corresponding platform files

(https://www.ncbi.nlm.nih.gov/geo/query/acc.cgi?acc=GPL10295) was used to map the gene Ids to genes. The averaged expression values were calculated and applied when multiple probes correspongding to a same gene for each sample. The probes which could't match any gene or matched not only a gene would be removed.

\section{Identification of MGPS in OS}

First, some metastases-related genes were obtained from CancerSEA (http://biocc.hrbmu.edu.cn/CancerSEA/), which is a database describes the functions of cancer cells [29]. We got 245 metastases-related genes and they would be used to follow analysis. The gene pair must have at least one metastases-related gene. Then, we built a 0-1 matrix for a pair of genes $\left(G_{m}\right.$ and $\left.G_{n}\right)$ in metastases and non-metastases OS patients. Lastly, if the frequency of metastases OS patients accord with a particular REO pattern was measured by fisher's exact test compared with non-metastases controls. The particular REO pattern was expression $G_{m}>G_{n}$ or $G_{m}<G_{n}$. The significant gene pairs evaluated with $\mathrm{P}<0.05$ were considered as MGPSs.

\section{Construction and analysis of metastases-specific co-expressed MGPS network and its topological features}

In order to construct a metastases-specific co-expressed MGPS network, pearson's correlation coefficients (PCCs) are calculated for each MGPS in metastases and non-metastases patients, respectively. The MGPSs were considered as metastases-specific co-expressed MGPSs, if absolute values of difference between PCCs in metastases and non-metastases patients were bigger than 0.2. Then a metastasesspecific co-expressed MGPS network was constructed using Cytoscape 3.3.0 (http://www.cytoscape.org/). The degree analysis of the network was also using Cytoscape 3.3.0.

\section{Survival analysis of MGPSs for OS patients}

In order to evaluate the performance about the MGPS for prognosis in OS, we performed survival analysis for genes in each MGPS. Follow the median value of expression level for each gene, the OS patients were divided into two risk groups. And then, Kaplan-Meier (K-M) survival analysis was used for the two groups. $\mathrm{P}<0.05$ was consider as prognostic gene for OS.

\section{Classification power of the prognostic MGPS in OS}

Consensus clustering approach was used to classify metastases and non-metastases OS patients based on expression data of genes [30]. A R package named ConsensusClusterPlus (https://www.r-project.org/) was performed to this process. Best category number was select when the areas of Cumulative 
distribution function (CDF) curves were smallest. Chi-square test was applied to evaluate if metastases and non-metastases OS patients could be classified using this method $(P<0.01)$.

\section{Functional and drug enrichment analysis for MGPSs in OS}

Online Enrichr (http://amp.pharm.mssm.edu/Enrichr/) tool was applied with default parameters to functional enrichment analysis for genes in MGPSs [31]. The enriched GO terms $(P<0.01)$ and KEGG pathways $(P<0.05)$ were extracted and considered as MGPS-associated functions. The gene-drug interaction data are download from DrugBank (https://www.drugbank.ca/) [32]. Then a drug-related MGPS network was constructed and analyzed to identify drug repurposing candidates for OS.

\section{Declarations}

\section{Funding}

Not applicable.

\section{Disclosure of interest}

The authors declare that they have no competing interest.

\section{Acknowledgments}

Not applicable.

\section{Availability of data and material}

The data that support the findings of this study are available from the corresponding author upon reasonable request.

\section{Ethics approval and consent to participate}

Not applicable.

\section{Patient consent for publication}

Not applicable.

\section{Competing interests}

The authors declare that they have no competing interests.

\section{Consent for publication}

Not applicable. 
Authors' contributions

ZGF conceived and designed the experiments, ZSR, LCB, FFG and LSH analysed the data, and ZYL, JCZ and ZBS wrote the manuscript.

\section{References}

1. Gonzalez-Fernandez Y, Brown HK, Patino-Garcia A, Heymann D, Blanco-Prieto MJ: Oral administration of edelfosine encapsulated lipid nanoparticles causes regression of lung metastases in pre-clinical models of osteosarcoma. Cancer Lett 2018, 430:193-200.

2. Negri GL, Grande BM, Delaidelli A, El-Naggar A, Cochrane D, Lau CC, Triche TJ, Moore RA, Jones SJ, Montpetit $A$ et al: Integrative genomic analysis of matched primary and metastatic pediatric osteosarcoma. J Patho/2019, 249(3):319-331.

3. Isakoff MS, Bielack SS, Meltzer P, Gorlick R: Osteosarcoma: Current Treatment and a Collaborative Pathway to Success. J Clin Oncol 2015, 33(27):3029-3035.

4. Huang $\mathrm{YM}$, Hou $\mathrm{CH}$, Hou SM, Yang RS: The metastasectomy and timing of pulmonary metastases on the outcome of osteosarcoma patients. Clin Med Oncol 2009, 3:99-105.

5. Jin K, Gao W, Lu Y, Lan H, Teng L, Cao F: Mechanisms regulating colorectal cancer cell metastasis into liver (Review). Oncol Lett 2012, 3(1):11-15.

6. Liang J, Chen M, Hughes D, Chumanevich AA, Altilia S, Kaza V, Lim CU, Kiaris H, Mythreye K, Pena MM et al: CDK8 Selectively Promotes the Growth of Colon Cancer Metastases in the Liver by Regulating Gene Expression of TIMP3 and Matrix Metalloproteinases. Cancer Res 2018, 78(23):6594-6606.

7. Hon CC, Ramilowski JA, Harshbarger J, Bertin N, Rackham OJ, Gough J, Denisenko E, Schmeier S, Poulsen TM, Severin $\mathrm{J}$ et al: An atlas of human long non-coding RNAs with accurate $5^{\mathbf{1}}$ ends. Nature 2017, 543(7644):199-204.

8. Tobin NP, Harrell JC, Lovrot J, Egyhazi Brage S, Frostvik Stolt M, Carlsson L, Einbeigi Z, Linderholm B, Loman N, Malmberg $\mathrm{M}$ et al: Molecular subtype and tumor characteristics of breast cancer metastases as assessed by gene expression significantly influence patient post-relapse survival. Ann Oncol 2015, 26(1):81-88.

9. Wang H, Sun Q, Zhao W, Qi L, Gu Y, Li P, Zhang M, Li Y, Liu SL, Guo Z: Individual-level analysis of differential expression of genes and pathways for personalized medicine. Bioinformatics 2015, 31(1):62-68.

10. Geman D, d'Avignon C, Naiman DQ, Winslow RL: Classifying gene expression profiles from pairwise mRNA comparisons. Stat App/ Genet Mol Biol 2004, 3:Article19.

11. Zhou X, Li B, Zhang Y, Gu Y, Chen B, Shi T, Ao L, Li P, Li S, Liu C et al: A relative ordering-based predictor for tamoxifen-treated estrogen receptor-positive breast cancer patients: multi-laboratory cohort validation. Breast Cancer Res Treat 2013, 142(3):505-514. 
12. Xu L, Tan AC, Winslow RL, Geman D: Merging microarray data from separate breast cancer studies provides a robust prognostic test. BMC Bioinformatics 2008, 9:125.

13. Aljubran AH, Griffin A, Pintilie M, Blackstein M: Osteosarcoma in adolescents and adults: survival analysis with and without lung metastases. Ann Oncol 2009, 20(6):1136-1141.

14. Li N, Jiang P, Du W, Wu Z, Li C, Qiao M, Yang X, Wu M: Siva1 suppresses epithelial-mesenchymal transition and metastasis of tumor cells by inhibiting stathmin and stabilizing microtubules. Proc Natl Acad Sci U S A 2011, 108(31):12851-12856.

15. Nakaya Y, Sukowati EW, Wu Y, Sheng G: RhoA and microtubule dynamics control cell-basement membrane interaction in EMT during gastrulation. Nat Cell Biol 2008, 10(7):765-775.

16. Rodriguez OC, Schaefer AW, Mandato CA, Forscher P, Bement WM, Waterman-Storer CM: Conserved microtubule-actin interactions in cell movement and morphogenesis. Nat Cell Bio/2003, 5(7):599609.

17. Kenific CM, Thorburn A, Debnath J: Autophagy and metastasis: another doubleedged sword. Curr Opin Cell Biol 2010, 22(2):241-245.

18. Langers I, Renoux VM, Thiry M, Delvenne P, Jacobs N: Natural killer cells: role in local tumor growth and metastasis. Biologics 2012, 6:73-82.

19. Phang JM, Liu W, Hancock CN, Fischer JW: Proline metabolism and cancer: emerging links to glutamine and collagen. Curr Opin Clin Nutr Metab Care 2015, 18(1):71-77.

20. Nguyen AN, Hollenbach PW, Richard N, Luna-Moran A, Brady H, Heise C, MacBeth KJ: Azacitidine and decitabine have different mechanisms of action in non-small cell lung cancer cell lines. Lung Cancer (Auckl) 2010, 1:119-140.

21. Zhang M, Yao C, Guo Z, Zou J, Zhang L, Xiao H, Wang D, Yang D, Gong X, Zhu J et al: Apparently low reproducibility of true differential expression discoveries in microarray studies. Bioinformatics 2008, 24(18):2057-2063.

22. Xiong G, Stewart RL, Chen J, Gao T, Scott TL, Samayoa LM, O'Connor K, Lane AN, Xu R: Collagen prolyl 4-hydroxylase 1 is essential for HIF-1alpha stabilization and TNBC chemoresistance. Nat Commun 2018, 9(1):4456.

23. Zhou Y, Jin G, Mi R, Zhang J, Xu H, Cheng S, Zhang Y, Song W, Liu F: Knockdown of P4HA1 inhibits neovascularization via targeting glioma stem cell-endothelial cell transdifferentiation and disrupting vascular basement membrane. Oncotarget 2017, 8(22):35877-35889.

24. Chakravarthi BV, Pathi SS, Goswami MT, Cieslik M, Zheng H, Nallasivam S, Arekapudi SR, Jing X, Siddiqui J, Athanikar J et al: The miR-124-prolyl hydroxylase P4HA1-MMP1 axis plays a critical role in prostate cancer progression. Oncotarget 2014, 5(16):6654-6669.

25. Cao XP, Cao Y, Li WJ, Zhang HH, Zhu ZM: P4HA1/HIF1alpha feedback loop drives the glycolytic and malignant phenotypes of pancreatic cancer. Biochem Biophys Res Commun 2019, 516(3):606-612.

26. Yunta M, Lazo PA: Apoptosis protection and survival signal by the CD53 tetraspanin antigen. Oncogene 2003, 22(8):1219-1224. 
27. Kuijjer ML, van den Akker BE, Hilhorst R, Mommersteeg M, Buddingh EP, Serra M, Burger $H$, Hogendoorn PC, Cleton-Jansen AM: Kinome and mRNA expression profiling of high-grade osteosarcoma cell lines implies Akt signaling as possible target for therapy. BMC Med Genomics 2014, 7:4.

28. Kuijjer ML, Peterse EF, van den Akker BE, Briaire-de Bruijn IH, Serra M, Meza-Zepeda LA, Myklebost O, Hassan $A B$, Hogendoorn $P C$, Cleton-Jansen AM: IR/IGF1R signaling as potential target for treatment of high-grade osteosarcoma. BMC Cancer 2013, 13:245.

29. Yuan H, Yan M, Zhang G, Liu W, Deng C, Liao G, Xu L, Luo T, Yan H, Long Z et al: CancerSEA: a cancer single-cell state atlas. Nucleic Acids Res 2019, 47(D1):D900-D908.

30. Wilkerson MD, Hayes DN: ConsensusClusterPlus: a class discovery tool with confidence assessments and item tracking. Bioinformatics 2010, 26(12):1572-1573.

31. Kuleshov MV, Jones MR, Rouillard AD, Fernandez NF, Duan Q, Wang Z, Koplev S, Jenkins SL, Jagodnik KM, Lachmann A et al: Enrichr: a comprehensive gene set enrichment analysis web server 2016 update. Nucleic Acids Res 2016, 44(W1):W90-97.

32. Wishart DS, Feunang YD, Guo AC, Lo EJ, Marcu A, Grant JR, Sajed T, Johnson D, Li C, Sayeeda Z et al: DrugBank 5.0: a major update to the DrugBank database for 2018. Nucleic Acids Res 2018, 46(D1):D1074-D1082.

\section{Figures}



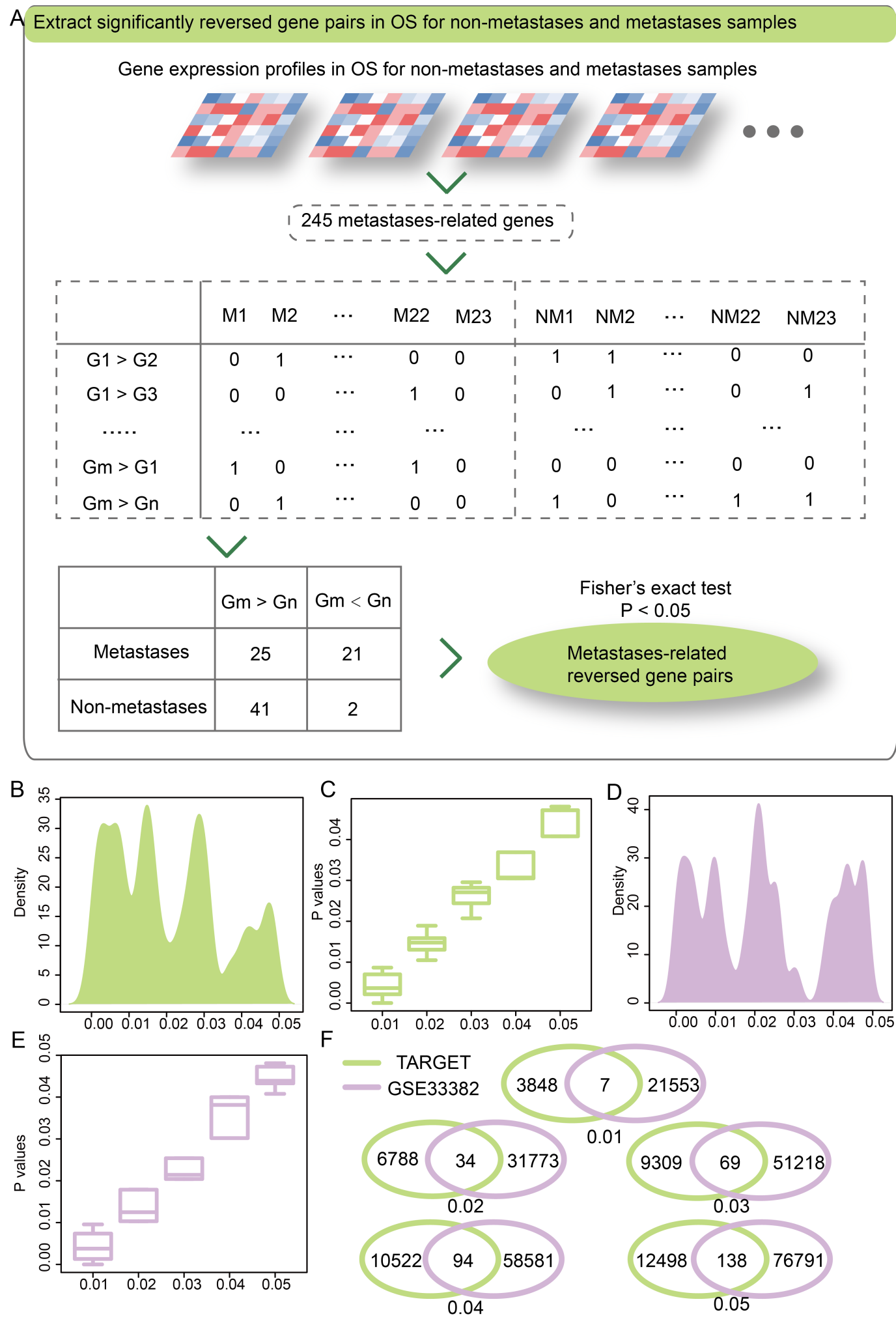

\section{Figure 1}

Identification of MGPSs based on gene expression profile in OS patients. (A) The workflow of identifying MGPSs based on gene expression profile in metastases and non-metastases samples. (B) The density distribution curve shows distribution of $P$ values of MGPSs based on TARGET dataset. (C) The box plots show $P$ values of MGPSs based on TARGET dataset in diverse regions. (D) The density distribution curve shows distribution of P values of MGPSs based on GSE33382 dataset. (E) The box plots show P values 
of MGPSs based on GSE33382 dataset in diverse regions. (F) The venn plots show intersection between TARGET and GSE33382 datasets follow diverse regions of $P$ values.
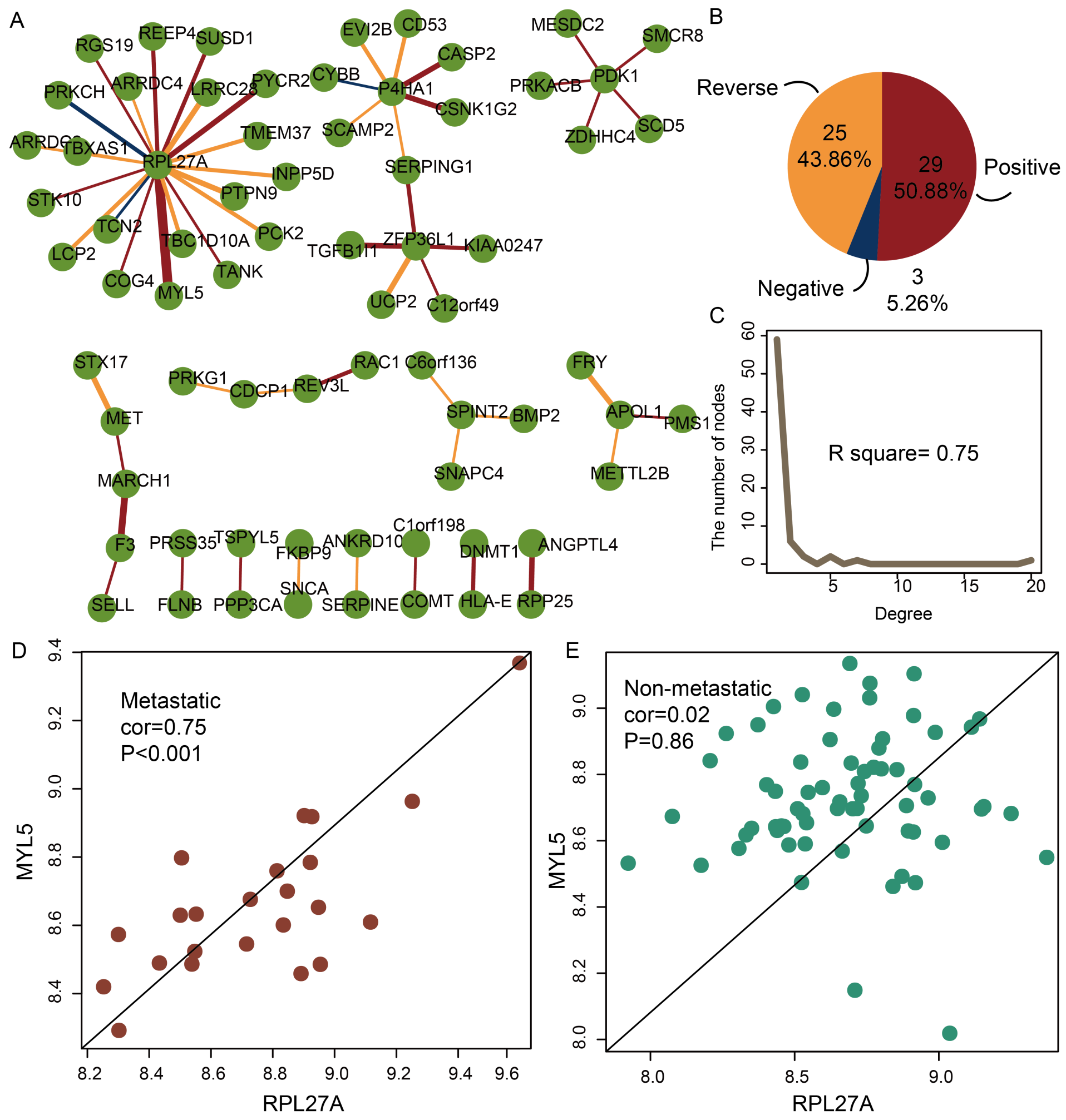

Figure 2

Construction of metastases-specific co-expressed MGPS network for OS. (A) A metastases-specific coexpressed MGPS network. Positive and negative correlations are represented by red and blue, respectively. The thicker edges represent bigger difference of correlations between metastases and non- 
metastases OS patients. (B) The pie chart shows percents for reverse, negative and positive interactions. (C) The plot shows degree distribution of metastases-specific co-expressed MGPS network. (D) The point plot shows the expression correlation of gene RPL27A and MYL5 in metastases samples. (E) The point plot shows the expression correlation of gene RPL27A and MYL5 in non-metastases samples.

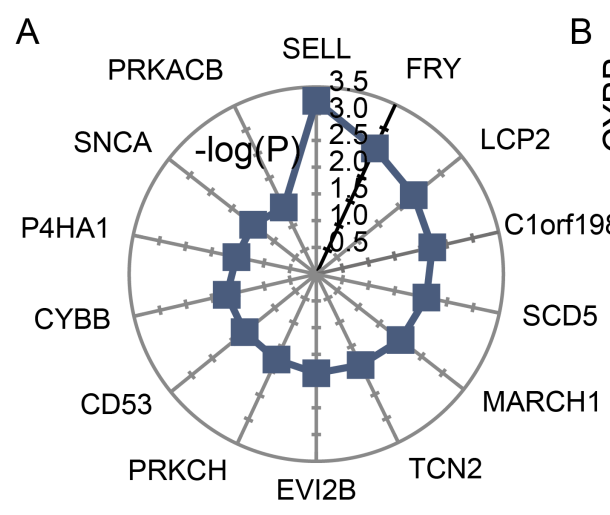

D Group Low Expression

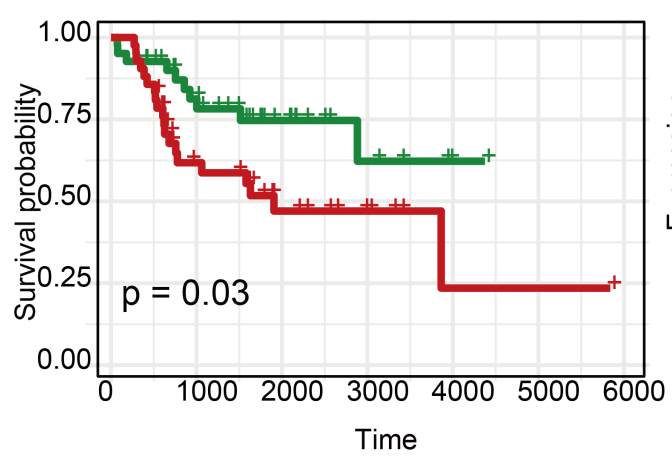

E

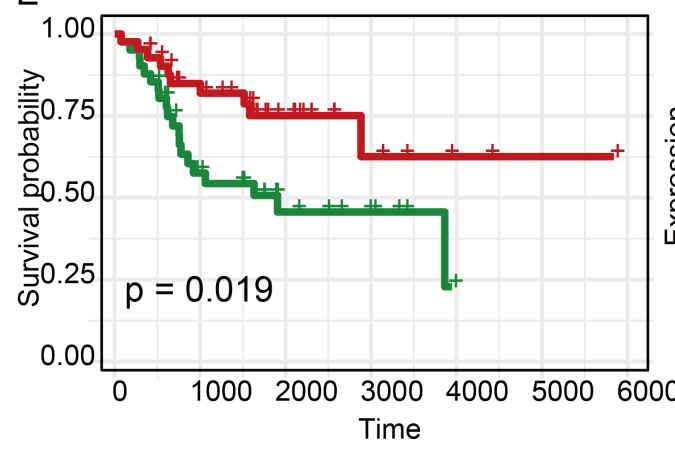

$\mathrm{F}$

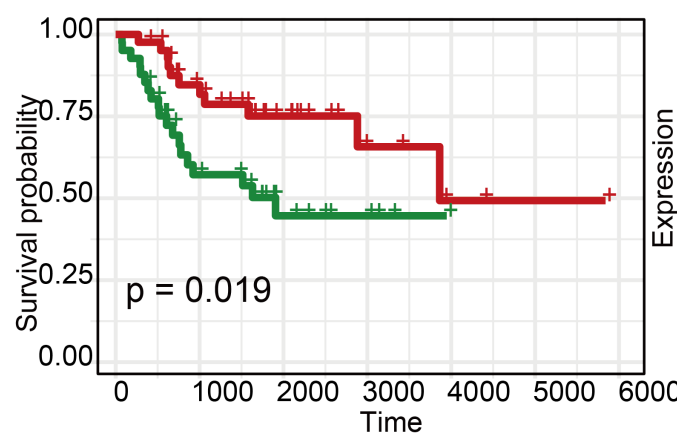

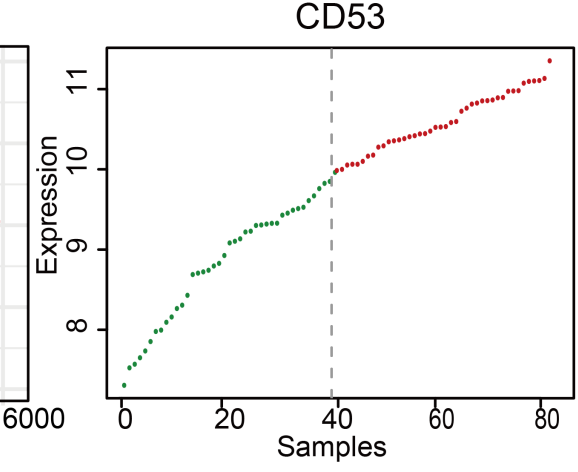

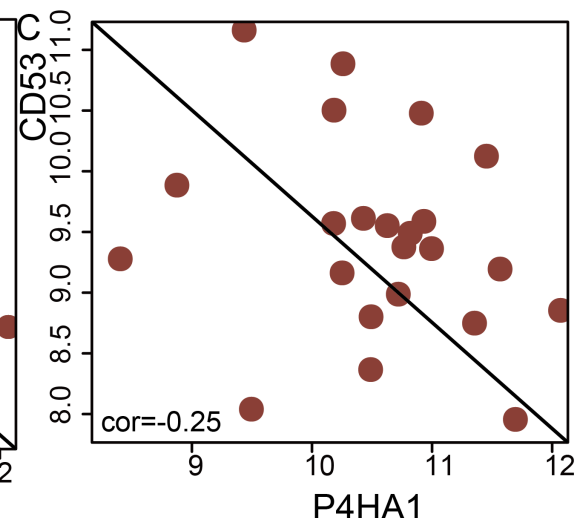

P4HA1

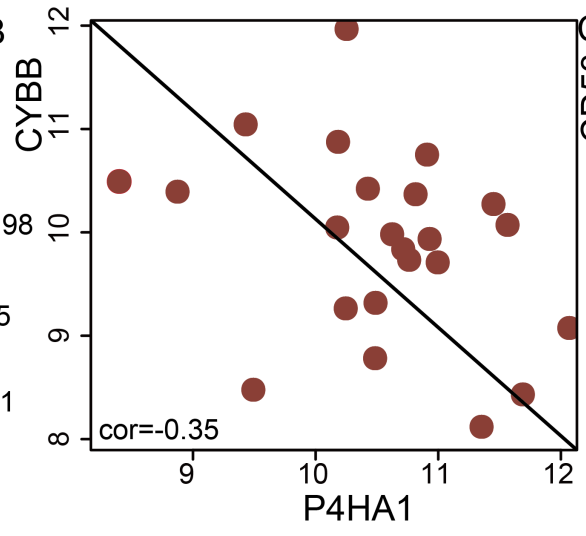

P4HA1
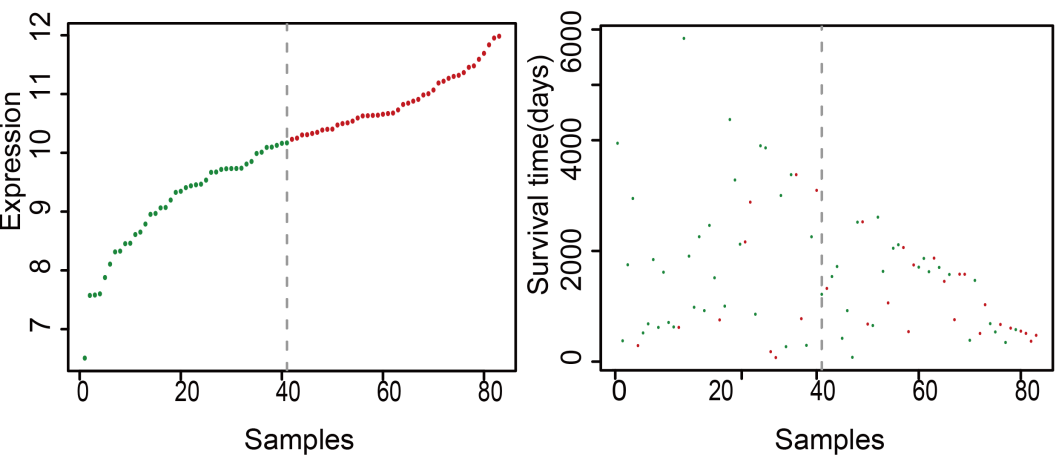

CYBB
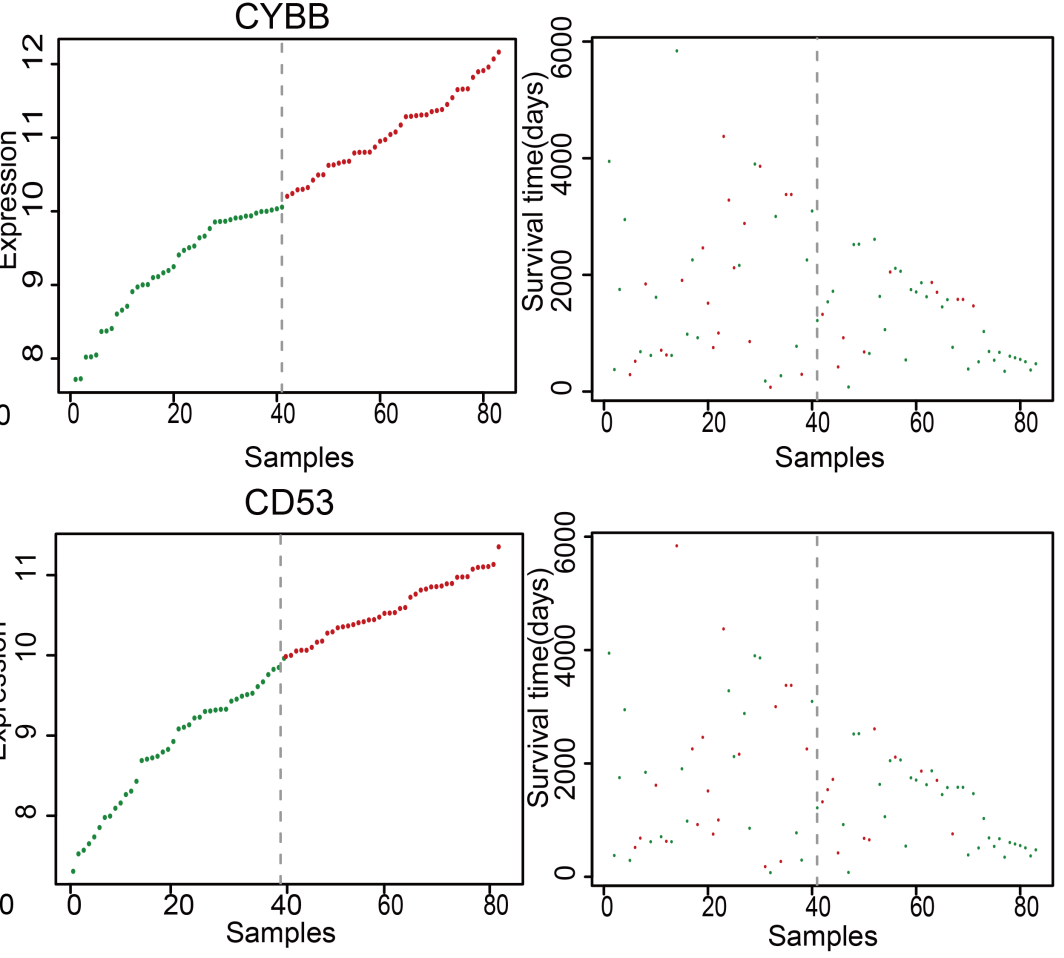

Figure 3 
Some MGPSs in metastases-specific co-expressed MGPS network were associated with survival in OS patients. (A) The radar chart shows the $P$ values of 13 prognosis-related MGPSs. (B) The point plot shows the expression correlation of gene P4HA1 and CYBB in metastases samples. (C) The point plot shows the expression correlation of gene P4HA1 and CD53 in metastases samples. (D-F) The overall survival of two OS groups with high and low expression are showed in K-M curves. Two-sided log-rank test is used to evaluate the difference between two K-M curves. The risk score distribution of the genes based on gene expression. The patient survival status of the genes.

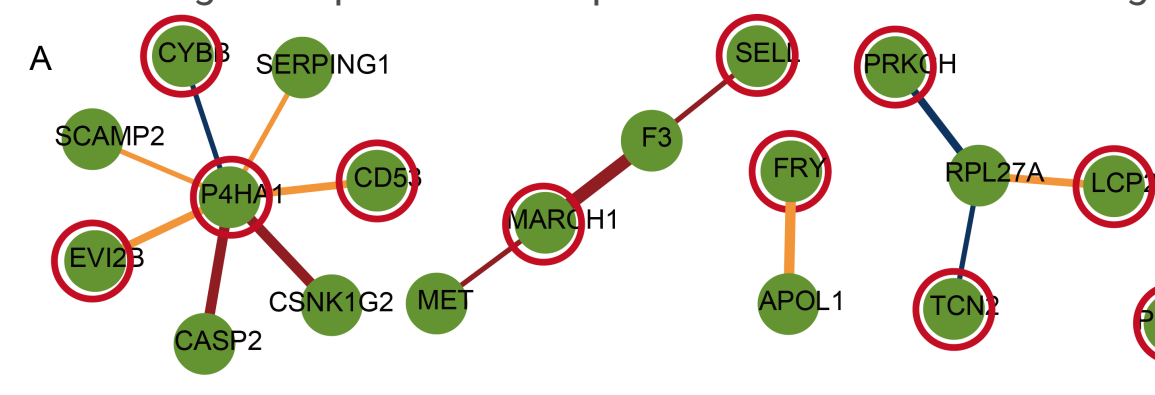

B

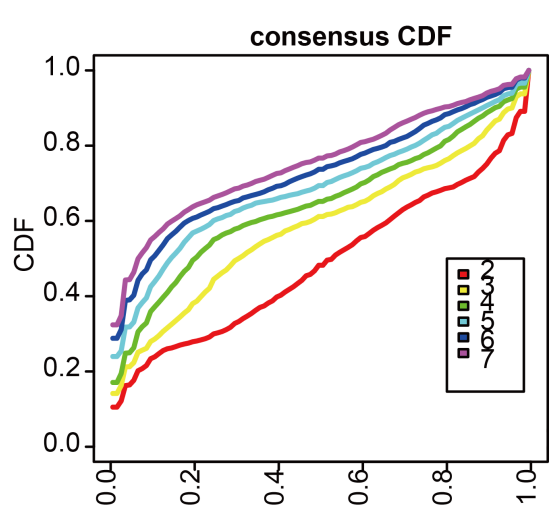

$\mathrm{E}$

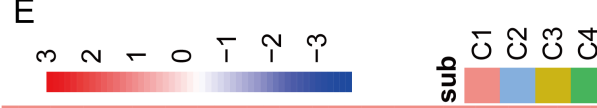

C
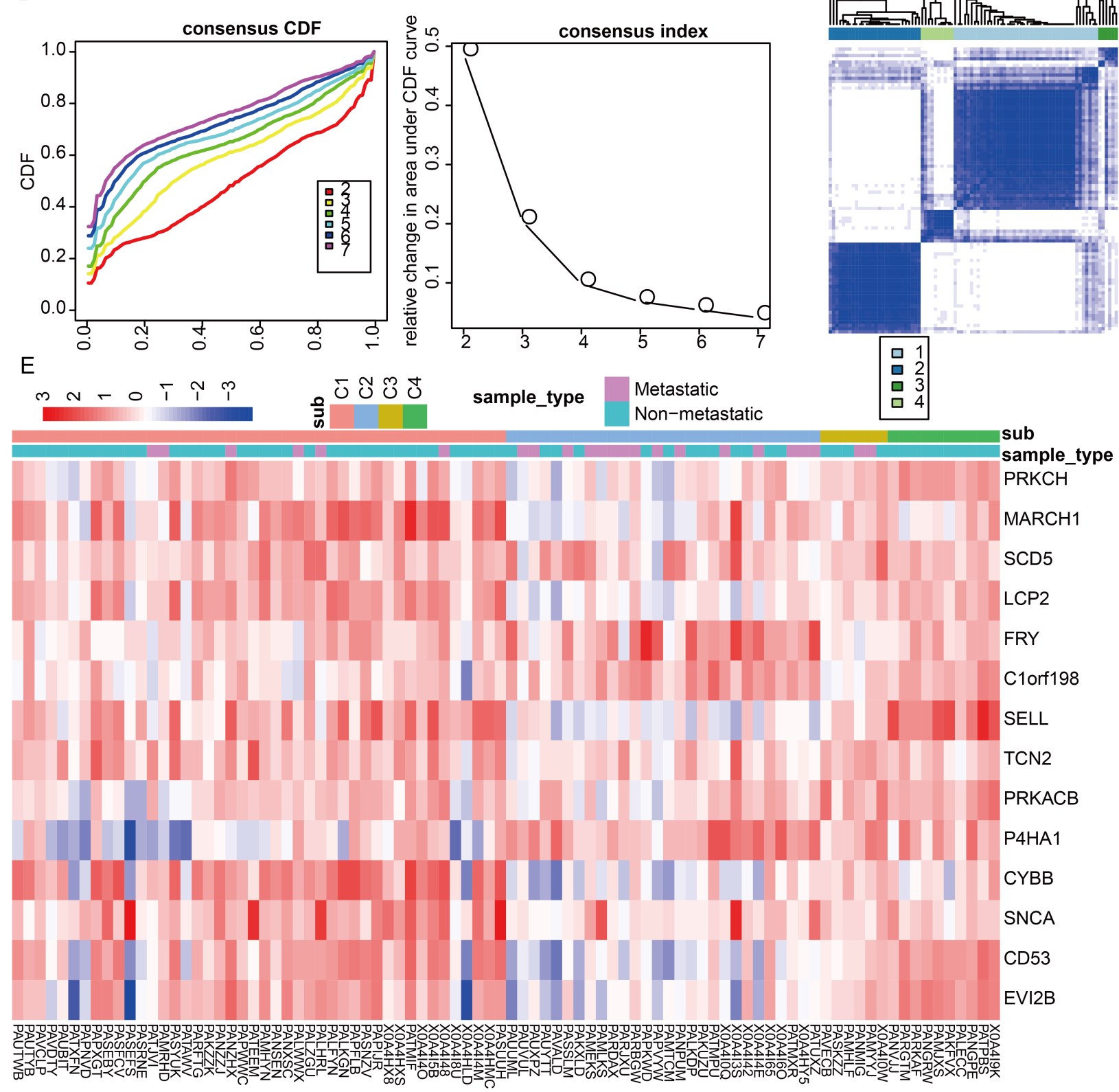


\section{Figure 4}

Prognosis-related genes in MGPSs could classify metastases and non-metastases OS patients. (A) Prognosis-related MGPS network for OS patients. The red circle represents prognosis gene. (B) Cumulative distribution function plot of the consensus index. (C) Relative change in area under CDF curve of different group number. (D) Consensus cluster heatmap of OS patients. (E) The gene expression heatmap, group type classified by consensus cluster is represented by sub label, the metastasis status of the OS patients is represented by sample type.

A

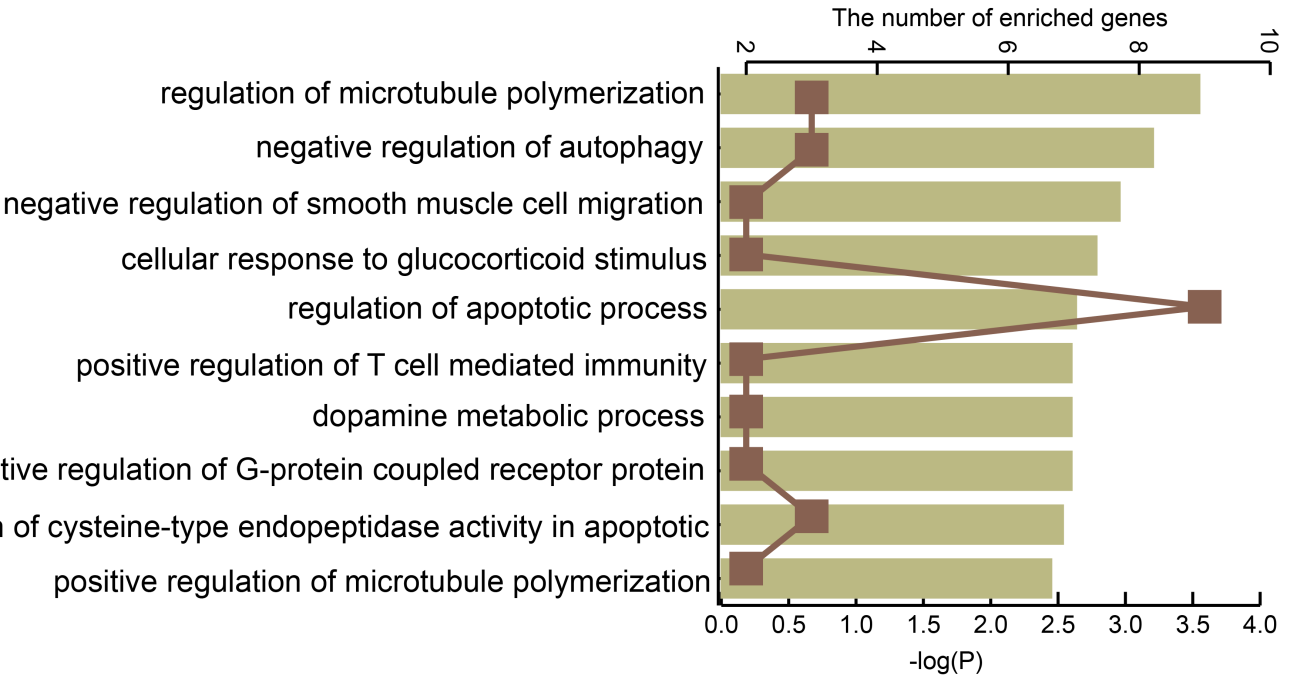

B

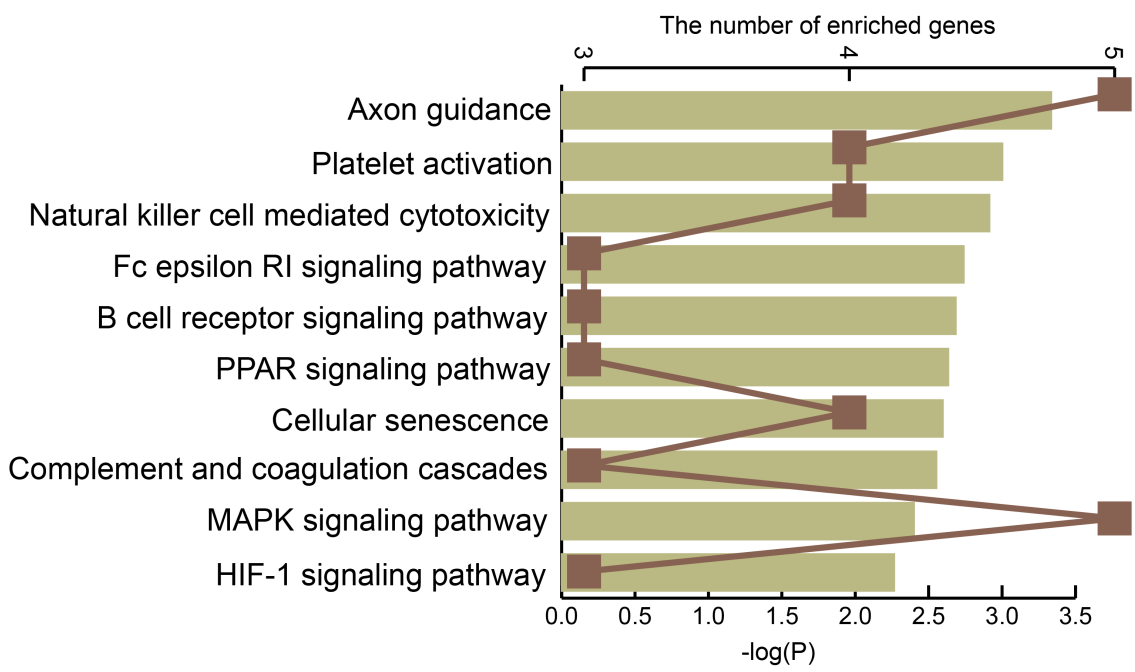

C
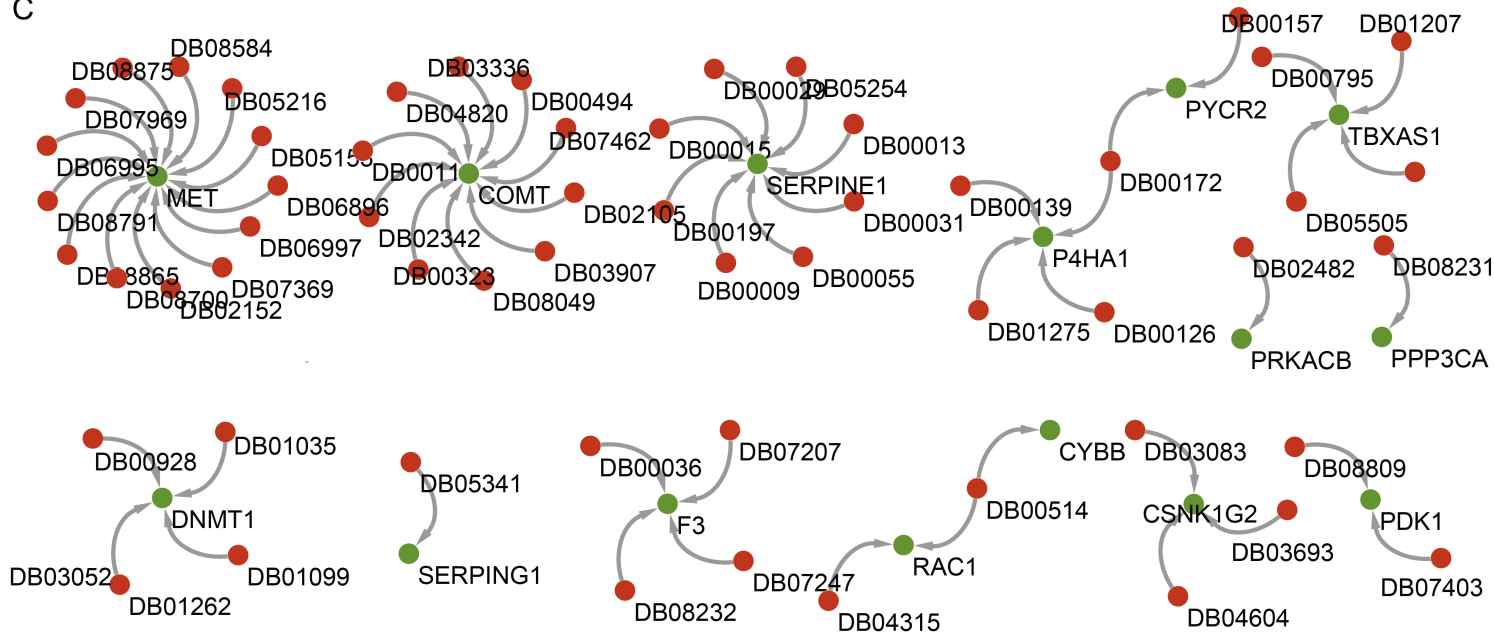

Page 16/17 


\section{Figure 5}

Functional characterizations and drug repurposing candidates of MGPSs in OS. (A) GO terms enriched for genes in MGPSs for OS and bar plots represent -log10(P). The dot line graph shows number of enriched genes in each GO term. (B) KEGG pathway enriched for genes in MGPSs for OS and bar plots represent $-\log 10(P)$. The dot line graph shows number of enriched genes in each KEGG pathways. (C) The drug and MGPS network. Red and green represent drug and gene, respectively. 